\title{
Wentzell Boundary Conditions in the Nonsymmetric Case
}

\author{
A. Favini $^{a 1}$, G. R. Goldstein ${ }^{b}$, J. A. Goldstein ${ }^{b}$ and S. Romanelli ${ }^{c}$ \\ ${ }^{a}$ Dipartimento di Matematica, Università degli Studi di Bologna, Piazza di Porta S. Donato 5 \\ 40126 Bologna, Italy \\ ${ }^{b}$ Department of Mathematical Sciences, University of Memphis, Memphis, Tennessee 38152 \\ ${ }^{c}$ Dipartimento di Matematica, Università degli Studi di Bari, via E. Orabona 4, 70125 Bari, Italy
}

\begin{abstract}
Let $L$ be a nonsymmetric second order uniformly elliptic operator with general Wentzell boundary conditions. We show that a suitable version of $L$ generates a quasicontractive semigroup on an $L^{p}$ space that incorporates both the underlying domain and its boundary. This extends the earlier work of the authors on the symmetric case.
\end{abstract}

Key words: Wentzell boundary conditions, nonsymmetric elliptic operators, $C_{0}$ semigroups, quasidissipative operators

AMS subject classification: 47D06, 47B44, 35K20

\section{Introduction}

In his study of the diffusion limit of the celebrated Wright-Fisher genetic model, W. Feller [4] showed that the limit of the corresponding $n$-state Markov chain was governed by a Markov process governed by the positive contraction $\left(C_{0}\right)$ semigroup on $C[0,1]$ with generator $A$ defined by

$$
\begin{gathered}
A u(x)=x(1-x) u^{\prime \prime}(x), \quad x \in[0,1], \quad '=\frac{d}{d x}, \\
D(A)=\left\{u \in C[0,1]: A u \in C[0,1], \quad \lim _{x \rightarrow j} A u(x)=0 \quad \text { for } j=0,1\right\} .
\end{gathered}
$$

Boundary conditions such as the one above (i.e. $A u=0$ on the boundary) were later introduced by A. D. Wentzell [10].

\footnotetext{
${ }^{1}$ Corresponding author. E-mail: favini@ dm.unibo.it
} 
Now, let

$$
L u:=\sum_{i, j=1}^{N} \frac{\partial}{\partial x_{i}}\left(a_{i j} \frac{\partial u}{\partial x_{j}}\right),
$$

where $x \in \Omega$, a $C^{2}$ bounded domain in $\mathbf{R}^{N},\left(a_{i j}(x)\right)$ is a uniformly positive definite real symmetric matrix in $C^{1}(\bar{\Omega})$, and the modulus of ellipticity is $\alpha_{0}>0$ :

$$
\sum_{i, j=1}^{N} a_{i j}(x) \xi_{i} \xi_{j} \geq \alpha_{0}|\xi|^{2}
$$

for all $x \in \bar{\Omega}$ and all $\xi \in \mathbf{R}^{N}$. The conormal derivative of $u$ with respect to $L$ at $x \in \partial \Omega$ is

$$
\frac{\partial^{L} u}{\partial \nu}:=\sum_{i, j=1}^{N} a_{i j}\left(\frac{\partial u}{\partial x_{j}}\right) \nu_{i}
$$

where $\nu=\left(\nu_{1}, \ldots, \nu_{N}\right)$ is the unit outer normal to $\partial \Omega$ at $x$. Of course, $\frac{\partial^{L}}{\partial \nu}$ reduces to the usual normal derivative $\frac{\partial}{\partial \nu}$ when $L$ is the Laplacian $\Delta$. The general Wentzell boundary condition for this problem is

$$
\alpha(x) L u+\beta(x) \frac{\partial^{L} u}{\partial \nu}+\gamma(x) u=0, \quad x \in \partial \Omega,
$$

where $\alpha, \beta, \gamma \in C^{1}(\partial \Omega), \alpha>0$ and $\beta>0$. Without loss of generality we may take $\alpha \equiv 1$. Let $A$ denote the operator $L$ with this boundary condition. Problems involving $A$ were studied on $C(\bar{\Omega})$ but not on $L^{p}(\Omega), p<\infty$, because $A$ is never quasi-dissipative on these $L^{p}$ spaces. (For semigroup background, see [1], [5].)

Beginning in [3] in 2002, we developed an $L^{p}$ theory for problems involving $A$. We showed, among other things, that the closure $A_{p}$ of a suitable realization of $A$ generates a $\left(C_{0}\right)$ quasicontractive semigroup on

$$
X_{p}=L^{p}(\Omega, d x) \oplus L^{p}\left(\partial \Omega, \frac{d S}{\beta}\right)=L^{p}(\bar{\Omega}, \mu)
$$

where

$$
\mu(E)=\int_{\Omega} \chi_{E}(x) d x+\int_{\partial \Omega} \chi_{E}(x) \frac{d S(x)}{\beta(x)}
$$

for $E$ a Borel set and $d S$ denotes the usual surface measure on $\partial \Omega$; here $1 \leq p<\infty$. We view $X_{p}$ as the closure of $C(\bar{\Omega})$ in the $X_{p}$ norm for $p$ finite; and we define $X_{\infty}=C(\bar{\Omega})$. The generation result mentioned above holds on $X_{p}$ for $1 \leq p \leq \infty$. Analyticity and other results hold as well; see [2] for the most recent results. We note that $A_{2}$ is self-adjoint and bounded above (by $\left\|\gamma_{-}\right\|_{\infty}$ ) on $X_{2}$.

The purpose of this paper is to present an analogue of the above result in the nonsymmetric case. We shall treat the uniformly elliptic case. 


\section{The main results}

As before, let $\Omega \subset \subset \mathbf{R}^{N}$ be a $C^{2}$ domain, $\mathcal{A}=\left(a_{i j}\right)$ a $C^{1}(\bar{\Omega})$ real symmetric $N \times N$ matrix function on $\bar{\Omega}$, and $\beta, \gamma$ real functions in $C^{1}(\partial \Omega)$ with $\beta>0$. Let $b \in C^{1}\left(\bar{\Omega}, \mathbf{R}^{N}\right)$ and $c \in$ $C(\bar{\Omega}, \mathbf{R})$. Suppose

$$
\sum_{i, j=1}^{N} a_{i j}(x) \xi_{i} \xi_{j} \geq \alpha_{0}|\xi|^{2}
$$

for some $\alpha_{0}>0$ and all $(x, \xi) \in \bar{\Omega} \times \mathbf{R}^{N}$. Let

$$
M u=\sum_{i, j=1}^{N} \frac{\partial}{\partial x_{i}}\left(a_{i j} \frac{\partial u}{\partial x_{j}}\right)+\sum_{i=1}^{N} b_{i} \frac{\partial u}{\partial x_{i}}+c u
$$

with boundary condition

$$
M u+\beta \frac{\partial^{L} u}{\partial \nu}+\gamma u=0, \quad \text { on } \quad \partial \Omega
$$

where $L$ is the symmetric version of $M$ corresponding to replacing each of $b, c$ by zero. Let

$$
D_{0}=\left\{u \in C^{2}(\bar{\Omega}): \quad(2.2) \text { holds }\right\}
$$

and let $A_{0}$ be the restriction of $L$ to $D_{0}$. Let

$$
X_{2}=L^{2}(\Omega, d x) \oplus L^{2}\left(\partial \Omega, \frac{d S}{\beta}\right) .
$$

Then, for all $u \in D_{0}$ and all $v \in C^{1}(\bar{\Omega})$,

$$
\begin{gathered}
<A_{0} u, v>_{X_{2}}=\int_{\Omega}(M u) \bar{v} d x+\int_{\partial \Omega}(M u) \bar{v} \frac{d S}{\beta} \\
=-\int_{\Omega} \sum_{i, j=1}^{N} a_{i j} \frac{\partial u}{\partial x_{j}} \frac{\partial \bar{v}}{\partial x_{i}} d x+\int_{\partial \Omega}\left(M u+\beta \frac{\partial^{L} u}{\partial \nu}\right) \bar{v} \frac{d S}{\beta}+\int_{\Omega} \sum_{i=1}^{N}\left(b_{i} \frac{\partial u}{\partial x_{i}}+c u\right) \bar{v} d x
\end{gathered}
$$

by the divergence theorem

$$
=-\int_{\Omega} \sum_{i, j=1}^{N} a_{i j} \frac{\partial u}{\partial x_{j}} \frac{\partial \bar{v}}{\partial x_{i}} d x-\int_{\partial \Omega} \gamma u \bar{v} \frac{d S}{\beta}+\int_{\Omega} \sum_{i=1}^{N}\left(b_{i} \frac{\partial u}{\partial x_{i}}+c u\right) \bar{v} d x
$$

by (2.2). In particular, for $u \in D_{0}$,

$$
\begin{gathered}
R e<A_{0} u, u>_{X_{2}} \leq-\alpha_{0} \int_{\Omega}|\nabla u|^{2} d x-\int_{\partial \Omega} \gamma|u|^{2} \frac{d S}{\beta} \\
+\operatorname{Re} \int_{\Omega}(b \cdot \nabla u) \bar{u} d x+\int_{\Omega} c|u|^{2} d x .
\end{gathered}
$$


To see why this holds, let $r=\operatorname{Re} u, s=\operatorname{Im} u$. Then $\nabla u=\nabla r+i \nabla s$ and

$$
\operatorname{Re}\left(\frac{\partial u}{\partial x_{j}} \frac{\partial \bar{u}}{\partial x_{k}}\right)=\operatorname{Re}\left(\frac{\partial r}{\partial x_{j}}+i \frac{\partial s}{\partial x_{j}}\right)\left(\frac{\partial r}{\partial x_{k}}-i \frac{\partial s}{\partial x_{k}}\right)=r_{x_{j}} r_{x_{k}}+s_{x_{j}} s_{x_{k}},
$$

whence, using obvious notation,

$\sum_{i, j=1}^{N} a_{i j} \frac{\partial u}{\partial x_{j}} \frac{\partial \bar{u}}{\partial x_{i}}=(\mathcal{A} \nabla u) \cdot \nabla \bar{u}=(\mathcal{A} \nabla r) \cdot \nabla r+(\mathcal{A} \nabla s) \cdot \nabla s \geq \alpha_{0}|\nabla r|^{2}+\alpha_{0}|\nabla s|^{2}=\alpha_{0}|\nabla u|^{2}$,

and (2.3) follows.

Next, by the Cauchy-Schwarz inequality, denoting by

$$
\|\nabla u\|_{L^{2}(\Omega)}^{2}=\int_{\Omega}|\nabla u|^{2} d x
$$

we have

$$
\operatorname{Re} \int_{\Omega}(b \cdot \nabla u) \bar{u} d x \leq\|b\|_{\infty}\|\nabla u\|_{L^{2}(\Omega)}\|u\|_{L^{2}(\Omega)} \leq\|b\|_{\infty}\left\{\epsilon\|\nabla u\|_{L^{2}(\Omega)}^{2}+\frac{1}{4 \epsilon}\|u\|_{L^{2}(\Omega)}^{2}\right\}
$$

for any $\epsilon>0$. Consequently,

$$
\begin{gathered}
R e<A_{0} u, u>_{X_{2}} \leq\left(-\alpha_{0}+\epsilon\|b\|_{\infty}\right)\|\nabla u\|_{L^{2}(\Omega)}^{2} \\
+\left\|\gamma_{-}\right\|_{\infty}\|u\|_{\left.L^{2}(\partial \Omega), \frac{d S}{\beta}\right)}^{2}+\left\|c_{+}\right\|_{\infty}\|u\|_{L^{2}(\Omega)}^{2}+\frac{\|b\|_{\infty}}{4 \epsilon}\|u\|_{L^{2}(\Omega)}^{2} \leq-\frac{\alpha_{0}}{2}\|\nabla u\|_{L^{2}(\Omega)}^{2}+k\|u\|_{X_{2}}^{2}
\end{gathered}
$$

provided $\epsilon>0$ is chosen so that

$$
\epsilon\|b\|_{\infty} \leq \frac{\alpha_{0}}{2}
$$

and thus

$$
k=\left\|\gamma_{-}\right\|_{\infty}+\left\|c_{+}\right\|_{\infty}+\frac{\|b\|_{\infty}}{4 \epsilon} .
$$

Here $w_{+}$(resp. $w_{-}$) is the positive (resp. negative) part of $w$. Thus $A_{0}$ is quasi-dissipative on $X_{2}$. The fact that $A_{0}-\lambda I$ has dense range for all sufficiently large real $\lambda$ follows from an application of the Lax-Milgram Lemma as in [3]. Of course in [3] the Lax-Milgram Lemma for symmetric sesquilinear forms was used; now we appeal to the nonsymmetric form (cf. [6], [7], [8], [9]). This completes the proof that $A_{2}$, the $X_{2}$-closure of $A_{0}$, is quasi-m-dissipative. It generates a positive semigroup since $\left(\lambda I-A_{2}\right)^{-1}$ is a positive operator for large $\lambda>0$. (See [1], [5].)

The extension to $X_{p}$, for $1<p<\infty$, follows the method used in [3], as does the extension to the limiting cases of $p=1, p=\infty$. Since no new ideas are necessary we omit the details. The following summarizes what we have just proved. 
Theorem 1. Let $\Omega$ be a bounded $C^{2}$ domain in $\mathbf{R}^{N}$, let $\left(a_{i j}(x)\right)$ be a real $N \times N$ symmetric uniformly positive definite matrix for $x \in \bar{\Omega}$, which is a $C^{1}$ function of $x \in \bar{\Omega}$. Let

$$
\begin{gathered}
b \in C^{1}\left(\bar{\Omega}, \mathbf{R}^{N}\right), \quad c \in C\left(\bar{\Omega}, \mathbf{R}^{N}\right), \\
\beta, \gamma \in C^{1}(\partial \Omega, \mathbf{R}), \quad \beta>0 .
\end{gathered}
$$

Define $M$ and $D_{0}$ by (2.1)-(2.2) and let $A_{0}$ be the restriction of $M$ to $D_{0}$. Let

$$
X_{p}=L^{p}(\Omega, d x) \oplus L^{p}\left(\partial \Omega, \frac{d S}{\beta}\right), \quad 1 \leq p<\infty,
$$

and $X_{\infty}=C(\bar{\Omega})$; and let $A_{p}$ be the $X_{p}$-closure of $A_{0}$.

Then $A_{p}$ is quasi-m-dissipative and generates a strongly continuous positive semigroup on $X_{p}$ for $1 \leq p \leq \infty$.

This semigroup is analytic, but we defer a precise statement of results of this type to a later paper.

\section{References}

1. K.-J. Engel, R. Nagel. One-Parameter Semigroups for Linear Evolution Equations. Graduate Texts in Math., Vol. 94, Springer-Verlag, Berlin, 2000.

2. A. Favini, G. R. Goldstein, J.A. Goldstein, E. Obrecht, S. Romanelli. Uniformly elliptic operators in divergence form and general Wentzell boundary conditions. (Submitted.)

3. A. Favini, G. R. Goldstein, J.A. Goldstein, S. Romanelli. The heat equation with generalized Wentzell boundary condition. J. Evol. Equ. 2 (2002), 1-19.

4. W. Feller. The parabolic differential equations and the associated semi-groups of transformations. Ann. of Math. (2) 55 (1952), 468-519.

5. J.A. Goldstein. Semigroups of Linear Operators and Applications. Oxford University Press, Oxford, 1985.

6. T. Kato. Perturbation Theory for Linear Operators. Springer Verlag, New York, 1966.

7. Z. M. Ma, M. Röckner. Introduction to the Theory of (Non-Symmetric) Dirichlet Forms. Springer-Verlag, Berlin, 1992.

8. E.M. Ouhabaz. Analysis of Heat Equations on Domains. Princeton University Press, 2004.

9. H. Tanabe. Equations of Evolution. Pitman Monographs and Studies in Math. London, San Francisco, Melbourne, 1979.

10 A.D. Wentzell. On boundary conditions for multi-dimensional diffusion processes. Theory Probab. Appl. 4 (1959), 164-177. 